\title{
MATEMATIZACIÓN: \\ LA NECESIDAD «REAL» DE LA FLUIDEZ EN LAS REPRESENTACIONES
}

\author{
LESH, R. \\ Universidad de Massachusetts-Dartmouth.
}

Este artículo se ha escrito como reacción al de Luis Rico y otros, acerca de los «Sistemas de representación y aprendizaje de estructuras numéricas". Mi artículo no puede considerarse una crítica, puesto que encuentro poco a criticar. En lugar de censurar, Io que me propongo hacer es destacar brevemente varios de los argumentos de Rico que considero de especial importancia y describir la otra cara de la moneda que no tuvo tiempo o espacio para desarrollar, pero que creo que es esencial en una discusión equilibrada acerca del papel que desempeñan las representaciones en el aprendizaje de las matemáticas y en lá resolución de problemas.

Los autores describen el núcleo central de su investigación diciendo: «Nuestro grupo de investigación está interesado en el estudio de las dificultades que los jóvenes encuentran en el manejo de las estructuras numéricas cuando se encuentran con nociones de matemática avanzada.» Después continúa describiendo interesantes representaciones concretas o gráficas que resultan útiles para afrontar las anteriores dificultades.
La investigación de Rico sigue estrechamente la tradición de otros estudiosos, incluido yo mismo, que han investigado los papeles que desempeñan las representaciones en el razonamiento matemático, centrándose en cómo Jos estudiantes dotan de sentido los problemas deI mundo o los cálculos simbólicos. Pero, en este artículo, me gustaría centrarme en un tipo de actividad matemática que tiende a insistir casi exactamente en el tipo opuesto de procesos necesarios para dotar de sentido a la mayoría de los cálculos simbólicos o los problemas verbales (ejercicios) que aparecen en los libros de texto, exámenes y enseñanzas tradicionales. Estas actividades implican la matematización de situaciones de la vida real.

En los problemas verbales (o en los ejercicios de cálculo) de los libros de texto tradicionales, los aspectos problemáticos tienden a incluir el esfuerzo para dar sentido a las preguntas planteadas simbólicamente; pero, cuanđo se intenta utilizar las matemáticas en situaciones de la vida real, los procesos necesarios subrayan, nor-

En las situaciones de la vida real en las que se utilizan esquemas matemáticos, los estudiantes realizan descripciones mąemáticas (simbólicas, gráficas) de situaciones que resultan significativas en sí mismas.

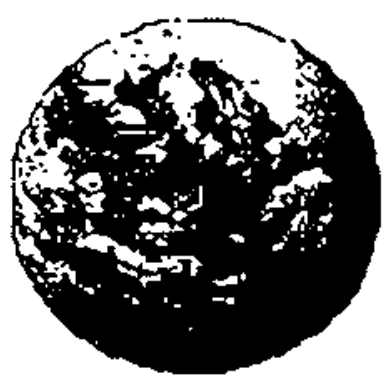

Munio real

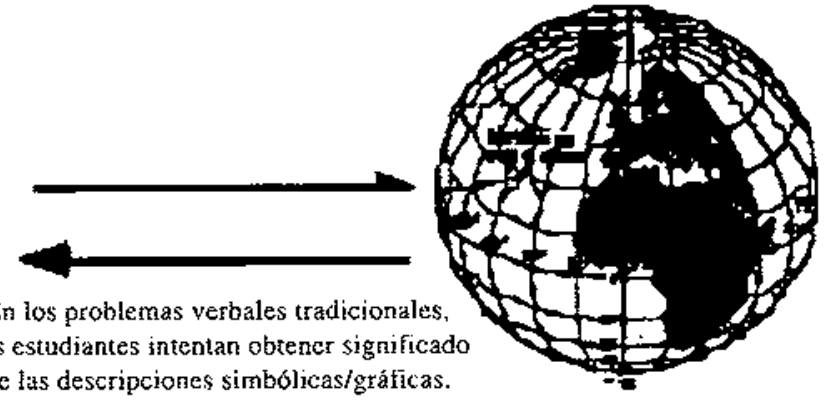

Modelo del mundo 
malmente, la necesidad de hacer descripciones simbólicas de situaciones que ya tienen sentido. Por consiguiente, con el fin de centrarnos en aquellas actividades en las que las capacidades de representación son preponderantes, este capítulo se va a centrar en aquellas actividades en las que los resultados que obtienen los estudiantes consisten, en gran parte, en descripciones, interpretaciones, explicaciones, construcciones o predicciones justificadas. Y, puesto que los productos que se espera de los estudiantes incluyen directamente las representaciones, la fluidez en crear representaciones desempeña un papel central en la comprensión y en las capacidades necesarias para el éxito.

\section{UN BREVE REPASO A ALGUNOS DE LOS PUNTOS CENTRALES DE RICO}

Entre las muchas perspectivas importantes que se describen en el artículo de Rico, me gustaría destacar las siguientes.

1) Ante todo, las matemáticas son el estudio de las estructuras. Contrariamente a las conclusiones a las que la mayoría ha llegado sobre la base de las experiencias escolares, las matemáticas no consisten simplemente en hacer lo que se te manda hacer, y el conocimiento matemático no es una simple lista de reglas mecánicas de acción-condición (definiciones, hechos o destrezas) que hay que programar dentro de la cabeza de los estudiantes y que se ejecutan sin fallos. Las matemáticas consisten en ver, al nuenos, tanto como en hacer.

$\mathrm{O}$, dicho de otro modo, hacer matemáticas implica más que la simple manipulación de símbolos matemáticos; implica interpretar situaciones matemáticamente; implica matematizar (o sea, cuantificar, visualizar o coordinar) sistemas estructuralmentc interesantes; implica utilizar un lenguaje especializado, símbolos, esquemas, gráficos, modelos concretos u otros sistemas de representación para desarrollar descripciones matemáticas, o explicaciones, o construcciones que permitan plantear predicciones útiles acerca de tales sistemas.

2) Cuando se construyen o se exploran sistemas matemáticos (estructuras), lo que tiene interés son las propiedades estructurales de esos sistemas, no los elcmentos aislados dentro del sistema, ni las reglas aisiadas que sirven para actuar sobre tales elementos. En consecuencia, cuando se generan representaciones para tratar dichas estructuras, lo que debe destacarse son las pautas y las regularidades que subyacen.

3) El significado de Ias estructuras matemáticas tiende a distribuirse a través de varios sistemas de representación que interactúan unos con otros, cada uno de los cuales destaca especialmente o ensombrece, en algún modo, características diferentes de las estructuras que subyacen.
Pues bien, en este artículo, me gustaría añadir el siguiente hecho a la lista anterior: los significados de las estructuras y de las representaciones matemáticas tienden a estar en interacción, a ser inestables y a evolucionar constantemente. Además, ilustraré por qué la interacción y la naturaleza inestable de las estructuras y representaciones matemáticas constituyen las fuerzas conductoras primarias que contribuyen a su evolución.

Para alcanzar el mencionado anterior, me centraré en los episodios de resolución de problemas de 60 minutos, a las que llamamos actividades de obtención de estructuras, puesto que el objetivo no consiste simplemente en ofrecer breves respuestas a las preguntas de otro; por el contrario, el objetivo implica desarrollar construcciones, descripciones, explicaciones o justificaciones que revelen explícitamente cómo se ha interpretado la situación. Por otro lado, también llamamos a estos episodios de resolución de problemas sesiones de desarrollo conceptual local, puesto que, con frecuencia, los ciclos de desarrollo estructural por los que atraviesan los jovenes para desarrollar sus respuestas resultan sorprendentemente semejantes a los estadios progresivos que los psicólogos del desarrollo han observado, a lo largo de periodos de varios años, para los sistemas conceptuaIes que subyacen tras estructuras matemáticas simples-pero-profundas, tales como las proporciones, las tasas de cambio, las fracciones o el razonamiento proporcional.

\section{LA MATEMATIZACIÓN IMPLICA MODE- LOS Y MODELIZACION}

Para ilustrar la diferencia entre matematizar y calcular, y también para ilustrar cómo ambos, matematización y cálculo, implican modelos matemáticos y modelización matemática, hemos utilizado el siguiente problema de las notas de examen con el fin de ayudar a los profesores de las escuelas elementales a entender lo que significa el uso de las operaciones aritméticas como modelos para describir situaciones de la "vida real» (Lesh, Amit y Schor, 1996).

Cuando estudiantes o profesores trabajan sobre el problema de las notas de examen, una de las cosas que tienden a reconocer es que la clasificación cambia drásticamente según la operación que se aplique para combinar las notas (adición, sustracción, multiplicacion, división, suma de vectores) o al aplicar algún otro procedimiento propuesto por los estudiantes (tal como las medias ponderadas). Por ejemplo, si se aplica la multiplicación, el orden de clasificación es: Ann, Bob, Dave, Carl, Gail, Emma, Fran, Herb e Ida (de menor a mayor). Pero si se aplica la división, el orden de clasificación pasá a ser: Carl, Bob, Fran, Ann, Emma, Ida, Herb, Dave y Gail. En otras palabras, cuando los estudiantes deciden qué operación(es) aplicar, están tomando automáticamente decisiones que tienen muchas más implicaciones. Tanto si se dan cuenta como si no, implícita o explícitamente, al decidir la operación a aplicar, automáticamente, tam- 


\section{EI Problema de las notas de examen}

Lea ef artículo de prensa siguiente. A continuación, escriba una carta al comité nombrado por el superintendente. Describa un procedimiento que usted les recomiende usar para resolver su problema. Utilice los datos de la tabla adjunta para demostrar cómo se puede utilizar su procedimiento para clasificar a los estudiantes, y explique por qué su procedimiento es mejor que otras ideas que se hayan podido proponer.

\begin{tabular}{|c|c|c|}
\hline Nombre & Calidad & Cantidad \\
\hline Ann & 8 & 8 \\
\hline Bob & 8 & 9 \\
\hline Carl & 8 & 10 \\
\hline Dave & 9 & 8 \\
\hline Emma & 9 & 9 \\
\hline Fran & 9 & 10 \\
\hline Gail & 10 & 8 \\
\hline Herb & 10 & 9 \\
\hline İda & 10 & 10 \\
\hline
\end{tabular}

bién tratan pares de notas completamente diferentes como si fueran equivalentes. Por consiguiente, para destacar de qué forma distintas operaciones se relacionan con distintas relaciones de equivalencia, la siguiente actividad resulta útil como continuación del problema de las notas de examen.

Si estadísticos profesionales trabajasen sobre el problema de las notas de examen, se referirian a las ecuaciones que plantearían en tanto que modelos matemáticos de ia situación. Se utiliza el término modelo debido a que las ecuaciones se basan en sistemas (o modelos) descriptivos de tipos diferentes que implican diferentes clases de cantidades y diferentes presunciones acerca de las relaciones que subyacen entre esas cantidades. Por ejemplo, el diagrama titulado clases de equivalencia para cinco operaciones básicas muestra resultados de la actividad de clases de equivalencia; y el diagrama tituiado la equivalencia depende de las operaciones muestra cómo un estudiante $S$ es equivalente a: a) el estudiante $D$ si se aplica la división para combinar las notas; $b$ ) el estudiante $M$ si se utiliza la multiplicación para combinar las notas; c) al estudiante $A$ si se utiliza la adición para

¿El comité escolar debate un sistema imparcial para combinar notas de examen!

Anoche, en la reunión mensual del comité escolar de nuestra ciudad, se suscitó una discusión acerca de cómo se debía clasificar la calidad del trabajo de arte de los estudiantes.

El departamento de arte no utiliza tests de respuestas breves para evaluar a los estudiantes. Por el contrario, cada estudiante realiza diez proyectos complejos a lo largo del año y se les dan dos notas por el conjunto de los proyectos.

Fred Wright, el director del departamento de arte, explicó el sjstema: «La nota de cantidad contempla los "objetivos de producto". Contamos cuántos proyectos ha terminado el estudiante satisfactoriamente. La nota de calidad contempla los "objetivos de proceso". Contamos la cantidad de herramientas y técnicas diferentes que ha utilizado el estudiante." Las discusiones empezaron cuando la administración intentó clasificar a los estudiantes según una escala única, del más bajo al más alto, de forma que se pudiera conceder premios especiales a los mejores estudiantes y los menos productivos se quedaran sin nominación.

El problema era que fos participantes en la reunión no podían ponerse de acterdo en cómo debran combinarse jas dos notas. Se propusieron cinco procedimientos distintos; y, al aplicar según qué procedimiento, los estudiantes aparecían clasificados más altos o más bajos.

a) Calidad + cantidad: El plan original consistía simplemente en sumar las dos notas, puesto que: «Eso es lo que se suele hacer con dos notas!»

b) Calidad - cantidad: En esta situación, la entrenadora de tenis dijo: «Cuando el tanteo de tenis es la suma, no nos importa cuántos puntos se han registrado para ilegar a ese punto.» Entonces propuso que podía ser lógico calcular la díferencia entre las dos notas, y dijo: «La calidad es lo que más nos deberia importar. Por tanto, deberiamos restar para climinar la calificación respecto a lá cantidad.»

c) Calidad x cantidad: A continuación, un hombre de negocios local sugirió que se podian multiplicar las dos notas para obtener una nota que signifique «calidad total». Pero, un profesor de física dijo que tambiên sería lógico dividir las notas para obtener una nota de calidad por unidad de cantidad (calidad/cantidad).

d) $\sqrt{\text {Calidad }^{2}+\text { cantidad }^{2}}$ : Finalmente, un profesor de matemáticas arguyó que calidad y cantidad representan dos dimensiones completamente distintas. Por tanto, se debería aplicar el teorema de Pitágoras para calcular la longitud del vector suma.

e) El especialista en exámenes de la escuela del distrito murmuró "¿A quién le importa cómo combinemos las dos notas mientras los resultados sean significativos en el nivel 0,5 ?»

Para cerrar la reunión de forma provechosa, el superintendente constituyó un comité que đebía presentar una recomendación acerca de cómo clasificar a los estudiantes mediante las notas disponibles.

combinar las notas; $d$ ) el estudiante $V$ si se utiliza la suma vectorial para combinar las notas; o e) otros estudiantes si se constituyen otros tipos de clases de equivalencia (que pueden no basarse en los resultados de una operacíon con números). No obstante, los estudiantes $D, M, A$ y $V$ se ordenan por sus resultados, de peor a mejor, dependiendo de qué operación se aplique.
Cuando los estudiantes o los profesores trabajan con el problema de las notas de examen y, después, en la actividad de las clases de equivalencia, los gráficos tienden a poner en evidencia que, cuando quienes resuelven los problemas deciden combinar las notas de examen aplicando alguna operación de cálculo (adición, sustracción, multíplicación o división ): a) no están sim- 
Actividad sobre "Clases de equivalencia»

(para realizarse después del problema de "Las notas de examen»)

1. Fn el problema "Las notas de examen", un estudiante cuyas notas de calidad y cantidad fucran 4 y 3 podría representarse gráficamentc mediante la coordenada $(4,3)$, en una gráfica como la que aquí se muestra.

a) Representar otros puntos en tos que la suma de las dos notas sca equivalente a la suma de $(4,3)$, b ) Utilizando los resultados de la parte $a$, trazar una línea (curva o recta, según convenga) que conecte los parcs de puntos que tengan la misma suma 1. c) Repetir los pasos $\theta$ y $b$ para otros puntos tales como: $(6,6)$ y $(6,8)$.

2. Repetir al problema 1 para cada una de las siguientes opcraciones: sustracción, multiplicación, división y suma vectorial.

' Falre las posibles respuestas encontramos: 4,7, 3,8, 8,3, 9,2 o cualesquicra otros puntos que estén en la diagonal que pasa por estos puntes.

plemente eligiendo un procedimiento para procesar los datos, sino que están adoptando también distintos sistemas descriptivos para describir la situación; $b$ ) estos sistemas descriptivos implican presunciones acerca de las relaciones y clases de equivalencia, así como procedimientos para operar con los datos; y c) estas presunciones, generalmente tácitas, tienen unas implicaciones enormemen:e importantes.

\section{ILA MODELIZACIÓN IMPLICA HACER PROPOSICIONES SIMBÓLICAS ACERCA DE SITUACIONES SIGNIFICATIVAS}

En la sección anterior, los problemas eran distintos a los problemas estcreotipados verbales de los libros de texto; pero seguían implicando darles un significado a situaciones planteadas simbólicamente (más que insistir en las actividades que plantean mayores exigencias acerca de las capacidades de representación). Por consiguiente, esta sección va a derivar su atención hacia problemas que impliquen directamente el desarrollo de descripciones matemáticas de situaciones significativas por sí mismas.

En el problema del avión de papel mostrado en la página siguiente, interviene la cuantificación en varios niveles. Primeramente, en un verdadero certamen entre aviones, cuando los estudiantes deciden cómo medir ciertas características, tales como el vuclo acrobático, el tiempo de planeo, la precisión, Ia durabilidad, e incluso la velocidad, lá cuantifícación de cierta información cuali-

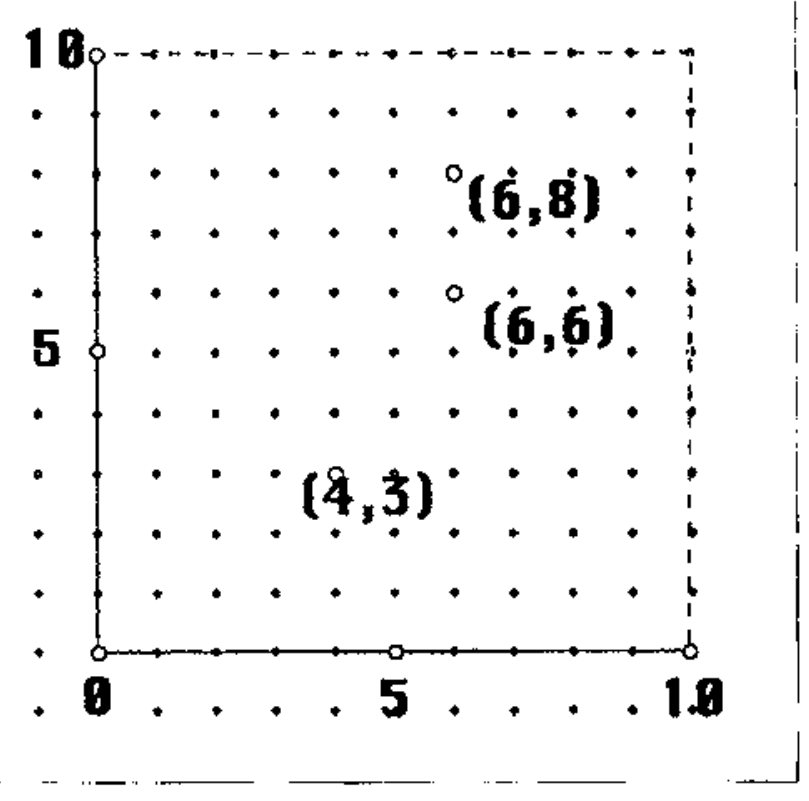

tativa es un factor que tiende a intervenir intensamente. En segundo lugar, incluso después de alcanzar un consenso acerca de cómo medir las anteriores características, se hacen necesarias las combinaciones de tales medidas con el fin de definir estructuras de orden superior, tales como «la calidad de conjunto», o «el más versátil». En tercer Iugar, cuando están implicadas varias cantidades, la calificación «bien» puede ser, para unos, una calificación alta, pero, para otros, «bien» puede ser una calificación baja; por tanto, los estudiantes pueden necesitar manejar cantidades con signo $(+/-)$ o cantidades direccionales; 0 , si las cantidades pucden no considerarse como equivalentemente importantes, se necesitará algún tipo de sumas ponderadas o de medias ponderadas.

En este capítulo, resulta conveniente abordar problemas como el problema del avión de papel por una serie de diferentes razones: $1^{\circ}$ ) Puesto que hacen hincapié en el proceso de matematización de situaciones significativas en sí mismas, esos problemas subrayan las capacidades de representación que tienden a obviarse en los problemas estereotipados verbales de los libros de tex to. $2^{\circ}$ ) En los casos en los que es posible hacer que los cstudiantes detecten la necesidad de una estructura como objetivo, los estudiantes, frecuentemente, la inventan (Lesh, Hoover y Kelly, 1993). Por consiguiente, este tipo de problemas permite a los investigadores ir más allá de las descripciones de estados estáticos de desarrolko para centrarse en los mecanismos que contribuyen al proceso de paso de un estado a otro. $3^{\circ}$ ) Cuando los estudiantes desarrollan las anteriores estructuras, a menudo lo hacen utilizando un valioso conjunto de representaciones inte- 
La equivalencia depende de las operaciones

Si $S=1,9 ; A=5,5 ; D=0,1 ; M=3,3 ;$ y $V=7,7$

Se tiene que:

$S=A$ aplicando la adición, puesto que $1+9=5+5$

$S=M$ aplicando la multiplicación, puesto que $1 \times 9=3 \times 3$

$S=D$ aplicando la división, puesto que $1 / 9 \approx 0 / 1$

$S=M$ apticando la suma yectrorial, puesto que $12+92 \approx 72 \times 72$

Nota: El símbolo ₹ significa: «Aproximadamente equivalente».

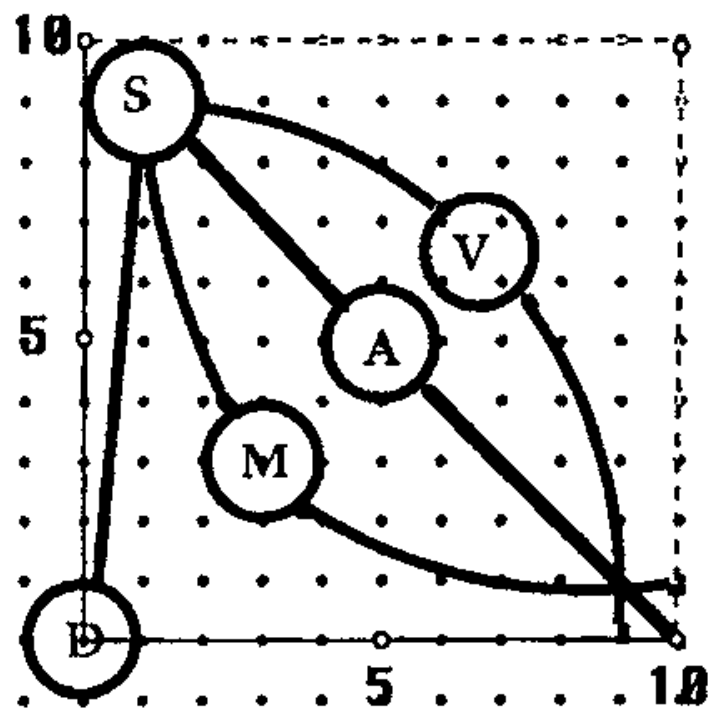

Clases de cquivalencia para cinco operáciones básicas

Clases de equivalencia para la ađición

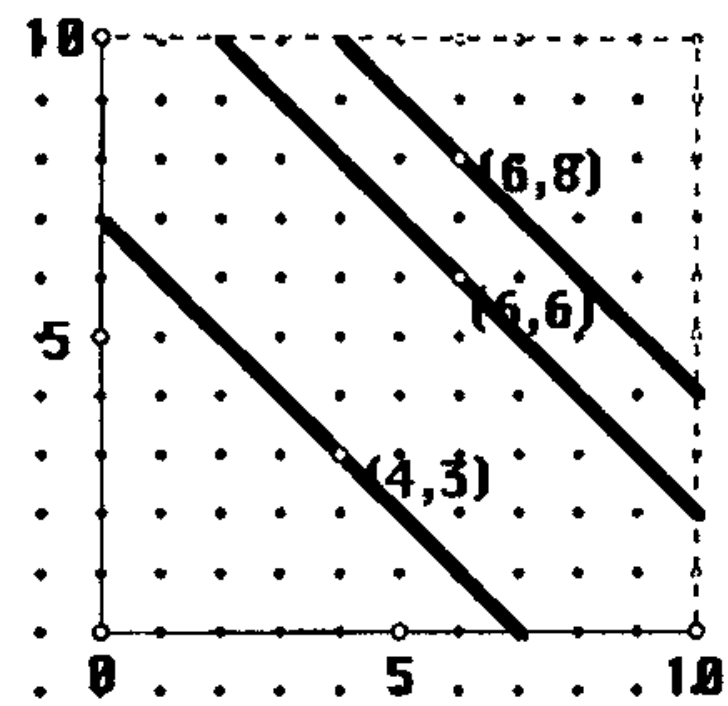

Clases de equivalencia para la sustracción

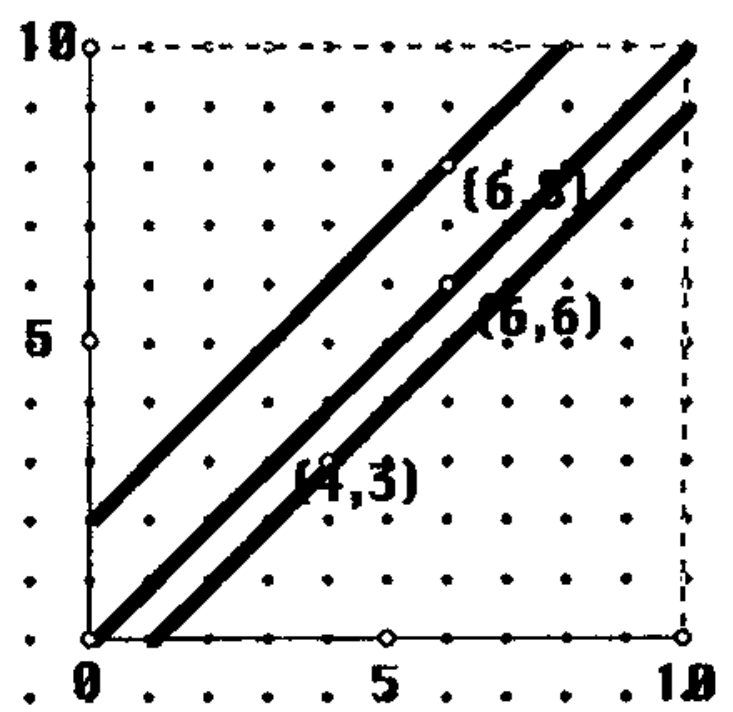

ractuantes, muchas de las cuales tienden a implicar diagramas iđiosincrásicos y sistemas de notación que Ios propios estudiantes han introducido. Además, a menudo, entre los estudiantes que son capaces de inventar estructuras potentes, Ios hay que han sido catalogados como poseedores de una «capacidad por debajo de la media», debido a su rendimiento en situaciones en las que intervienen exámenes, libros de tex to y una enseñan- 
Clases de equivalencia para la multiplicación

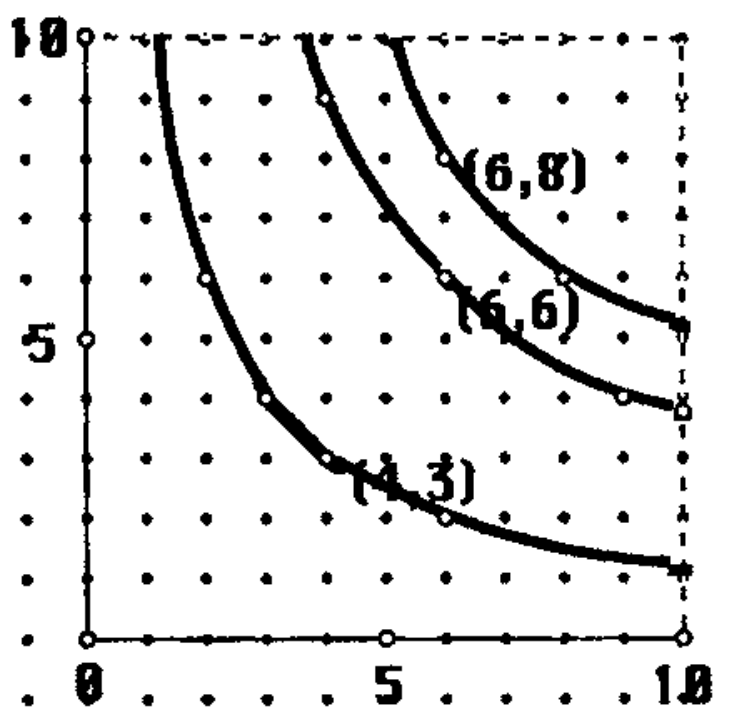

Clases de equivalencia para la suma vectorial

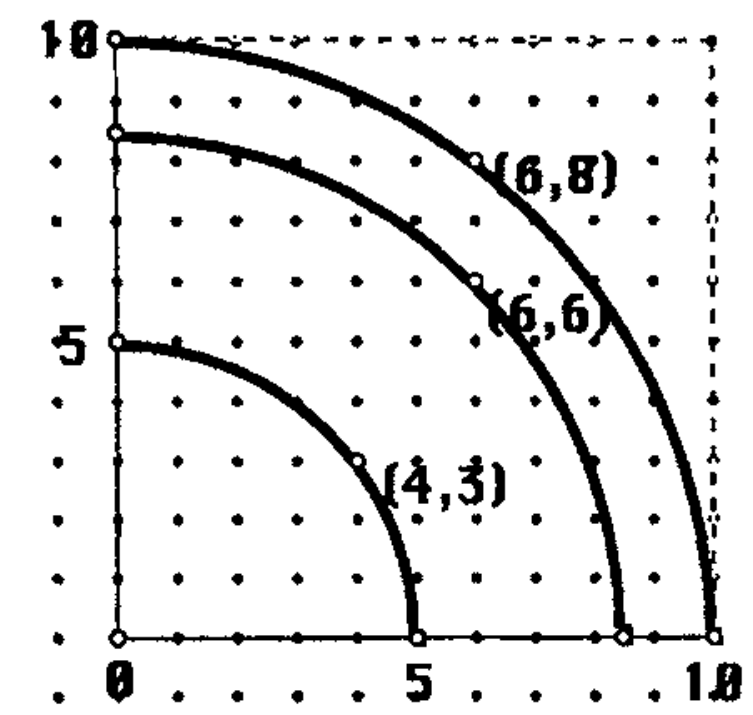

Clases de cquivalencia para la división

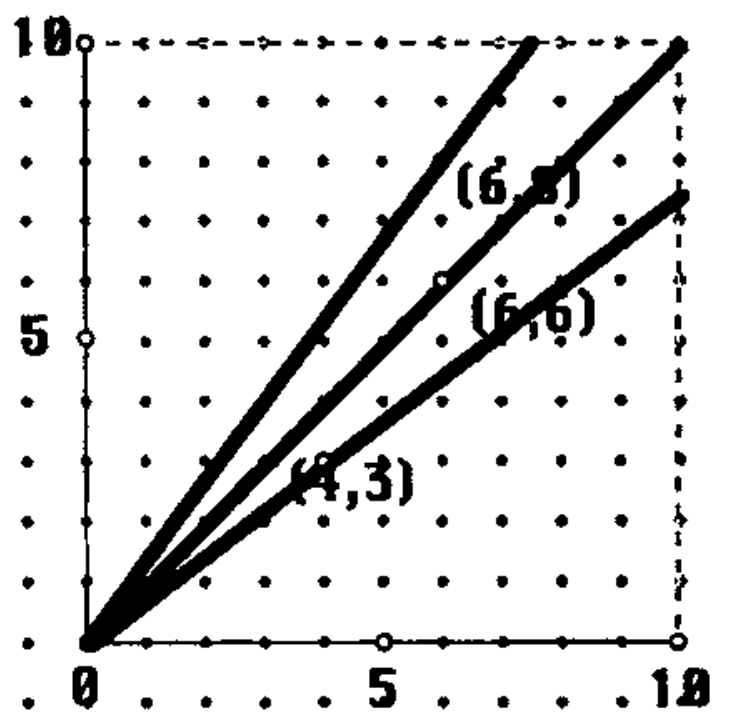

Clases de equivalencia sin operación alguna

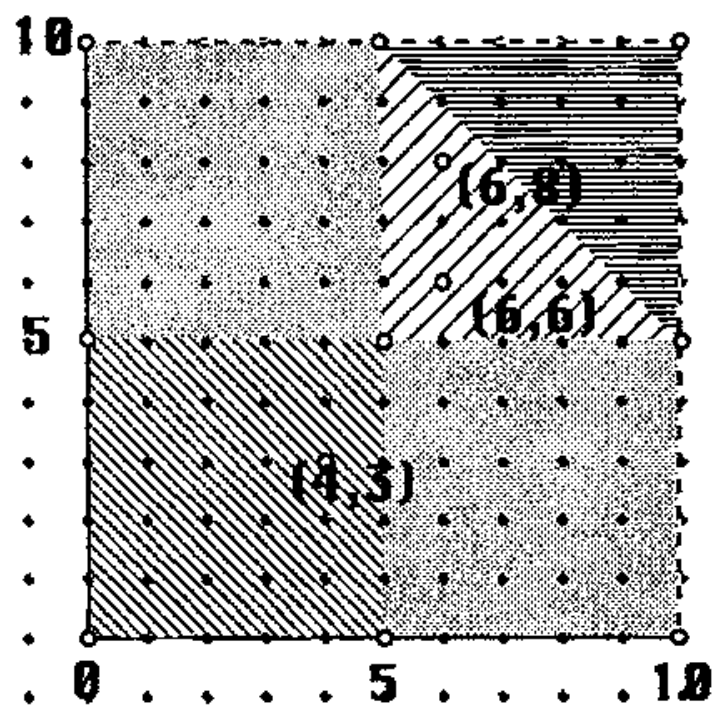

7a tradicionales, en las que se insiste solamente en una gama estrecha y poco profunda de capacidades matemáticas (Lesh, Hoover y Kelly, 1993). 4º Experiencias como la del problema del avión de papel proporcionan «relatos» (o experiencias) que, a menudo, funcionan de forma simbólica. Es decir, que los estudiantes utilizan estas experiencias como prototipos para pensar acerca de otras situaciones similares de resolución de problemas (Schank, 1990).
Uno de los puntos en los que el problema del avión de papel permite insistir es en que el tipo de cantidades, de relaciones y de operaciones necesarias en situaciones cotidianas comprende mucho más que las simples cuentas, medidas y formas que ocupan un alto porcentaje det currículo de matemáticas desđe la escuela infantil hasta COU en los Estados Unidos. Los estudiantes necesitan también: clasificaciones, coordenadas, cantidades direccionales y con signo, informacion cualitativa cuan- 


\section{El problema del avión de papel}

Para el contexto del avión de papel descrito a continuación, escriba una carta a los jueces en la que se describa cómo deben utilizarse las calificaciones para calcular el ganador de cada prueba. Después, describa cómo especificar un ganador general para ambas pruebas juntas.

Nota: Para garantizar que este problema implica la matematización de una situación significativa, resulta útil basar el problema en un verdadero certamen de aviones que lleven a cabo los estudiantes, en lugar de basarlo en una narración acerca de un certarnen de aviones, como hacemos en la descripción del problema dada a continuación.

\section{El gran certamen de aviones de papel}

Como parte de la feria científica de este año, los estudiantes de séptimo grado de la Escuela Jefferson realizaron un certamen de aviones de papel. Para preparar el campeonato, los estudiantes realizaron experimentos de tal modo que independientemente de los diferentes propósitos que se plantearon, e independientemente del tamaño y tipo de papel que se les proporcionó, podian diseñar un avión con las siguientes características:

* capacidad para aterizar cerca de una diana concreta (precisión)

* capacidad para mantenerse en vuelo (volar despacio) frente a la capacidad de volar rápido

* capacidad para volar lejos

* capacidad para volar acrobáticamente (con muchos giros, vueltas y cspirales)

* capacidad para realizar varios vuelos sin necesidad de reparaciones o reconstrucciones (durabilicad)

El dja del certamen, a cada equipo se le proporcionó una hoja de papel de máquina de escribir. A continuación, sin permitírseles ninguna prucba previa, los estudiantes diseñaron sus aviones y los lanzaron desde un punto de partida señalado en el suelo del gimnasio. Se realizaron dos pruebas. Una centrada en el tiempo de vuelo y precisión, y la otra en la velocidad y precisión.

\section{Prueba del tiempo de vuelo}

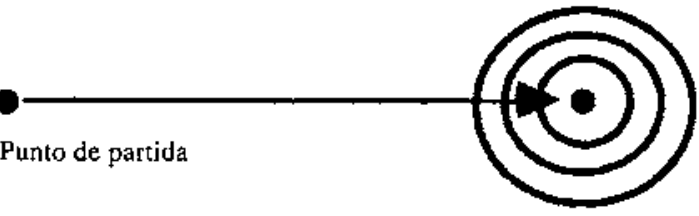

\section{Punto de partida}

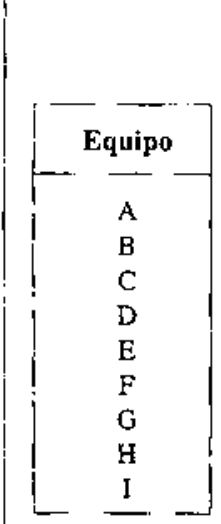

\begin{tabular}{|c|c|c|c|}
\hline \multicolumn{2}{|c|}{ Tiempo de vuelo y precisión } & \multicolumn{2}{|c|}{ Velocidad y precisión } \\
\hline Tiempo & Distancia & Tiempo & Distancia \\
\hline 3 segundos & 1 metro & 3 segundos & 1 metro \\
4 segundos & 1 metro & 4 segundos & 1 metro \\
5 segundos & 1 metro & 5 segundos & 1 metro \\
3 segundos & 1 metro & 3 segundos & 1 metro \\
4 segundos & 2 metros & 4 segundos & 2 metros \\
5 segundos & 2 metros & 5 segundos & 2 metros \\
3 segundos & 3 metros & 3 segundos & 3 metros \\
4 segundos & 3 metros & 4 segundos & 3 metros \\
5 segundos & 3 metros & 5 segundos & 3 metros \\
\hline
\end{tabular}

Para la prueba de tiempo de vuelo y precisión, los estudiantes tenían que lanzar sus aviones desde el punto de partida hasta un punto que estaba senalado en el suelo a treinta pies de distancia. El objetivo consistía en acercarrse lo más posible a la diana y en mantenerse en vuelo el mayor tiempo posible.

Punto de partida

Para la prueba de velocidad y precision, tos estudiantes tenian que lanzar sus aviones desde el punto de partida, doblando una esquina, hasta un punto que estaba señalado en el suelo a treinta pies de distancia De nuevo, el objetivo consistía en acercarse lo más posibie a la diana, pero el avión debía llegar lo más rápidamente posible.

La tabla siguiente muestra los resuitados de ambas pruebas. Las calificaciones de «tiempo» muestran el tiempo que el avión permaneció en el aire: 1 unidad, 2 unidades y 3 unidades. "Distancia» registra lo cerca que atertizó cada avión de la diana: 1 metro, 2 metros o 3 metros.
Prueba de velocidad 
tificada, cantidades acumulativas, cantidades en constante cambio, transformaciones, pautas o características de conjuntos de datos (más que porciones aisladas de datos), u otros tipos de sistemas para cuya descripción son útiles las estructuras matemáticas elementales. En cambio, en los Estados Unidos, cuando la mayoría de los estudiantes acaban el bachillerato de matemáticas, incluso los que lo hacen con los mejores resultados, muy pocas veces se han visto estimulados a desarrollar más allá de uná exigua colección de modelos y representaciones que sirven para dotar de sentido a los sistemas en los que los «objetos» tienden a quedar restringidos a simples cuentas, medidas y formas, a los que se añade una cstrecha y corta colección de razones y tasas o proporciones.

\section{REPRESENTACIÓN: SIMPLIFICACIÓN DE SISTEMAS EXTERNOS O EXTERNALI- ZACION DE SISTEMAS INTERNOS}

En las anteriores secciones, se han tratado las representaciones como si fueran fundamentalmente externalizaciones de sistemas internos de pensamiento. Pero, cuando los estudiantes utilizan representaciones para matematizar situaciones de resolución de problemas, las representaciones matemáticas funcionan como simplificaciones de sistemas externos, al menos tanto como externalizaciones de sistemas internos. Para ver un ejemplo en el que se tiende a destacar esta última función, consideremos el problema de los empleos veraniegos, dado en la página siguiente (Katims, Lesh et al., 1994).

Tanto el problema de los empleos veraniegos como el problema del avión de papel son actividades de obtención de estructuras. En cada uno de ellos, el objetivo consiste en generar una definición operativa que estipule el concepto que tiene el resolutor del problema de cómo medir una 〈listribución (tal como la «productividad» de los empleos veraniegos, o la «capacidad de planear» de los aviones de papel). Por tanto, las descripciones, las explicaciones y las justificaciones no son simples comparsas de las respuestas aplicables, son el núcleo de dichas respuestas aplicables. Además, puesto que se pretende que el problema de los empleos veraniegos sca abordado por equipos de tres estudiantes de enseñanza media con una capacidad normal, tiende a plantear fuertes exigencias en cuanto a la capacidad de comunicación y fluidez en la representación, con los siguienies objetivos: a) analizar problemas y planificar soluciones que impliquen mútitiples pasos y múltiples recursos y restricciones; b) justificar y explicar las acciones propuestas, y predecir sus consecuencias; $c$ ) gestionar y evaluar cl proceso; $d$ ) integrar y comunicar los resultados de forma que resulten útiles a los demás.

El problema de los empleos veraniegos está pensado para que st: le dediquen, al menos, una o dos clases completas (suponiendo que se han planteado cuestiones preparatorias y que se cuenta con los elementos adecuados como calculadoras y papel milimetrado). Por consiguiente, puesto que el desarrollo de estructuras es de lo que se trata exclusivamente en las soluciones de este tipo de problemas, y puesto que esta evolución tiende a implicar una serie de ciclos de modelización en los que se introducen, se comprueban y se afinan representaciones y modos de pensamiento cada vez más sutiles, el objetivo de las representaciones no consiste simplemente en que los estudiantes se comuniquen entre sí, sino que los estudiantes se comuniquen comsigo mismos y que externalicen sus propios modos de pensamiento de forma que puedan ser examinados y mejorados. Por lo tanto, los significados y funciones de las representaciones que aplican los estudiantes no son estáticos y están continuamente evolucionando; y es cierto también para las estructuras subyacentes implicadas, así como para los sistemas externos que describen.

Pondremos algunos ejemplos:

Algunas características de las primeras interpretaciones de los estudiantes

Las primeras interpretaciones consisten frecuentemente en un «batiburrillo» de diversos modos de pensamiento desorganizado $y$, a veces, inconsistente, acerca de los datos, de los objetivos y de los pasos hacia una posible solución. Pongamos por caso, si trabajan tres personas en grupo, a menudo no consiguen darse cuenta de que cada uno está: a) pensando acerca de la cuestión de forma diferente, $b$ ) centrándose en informaciones y relaciones distintas, c) apuntando a objetivos, en cierto modo, diferentes, y $d$ ) considerando distintos procedimientos para alcanzar aquellos objetivos. De hecho, incluso para un solo individuo, el estudiante puede no conseguir mantener una interpretación consistente y saltará, inconscientemente, de uno a otro modo de pensar sin darse cuenta del cambio que ha hecho. Puesto que los problemas como el problema de los empleos veraniegos frecuentemente contienen tanto «demasiada» como "insuficiente» información, las primeras representaciones y modos de pensamiento de los estudiantes suelen centrarse solamente en un subconjunto de dicha información y suelen tender a preocuparse de encontrar formas de simplificar o de añadir (suma, media) toda la información disponible. Por ejemplo, en su primera interpretación del problema de los empleos veraniegos, con frecuencia, Ios estudiantes se centran en las recaudaciones, descuidando los horarios de trabajo; o se centran en los periodos de ventas fáciles, ignorando los periodos bajos o normales; o fijan su atención solamente en un mes, dejando de lado tos otros dos meses. Sin embargo, al mismo tiempo que los estudiantes se preocupan por encontrar modos de simplificación o de reducción de la información, pueden también expresar su inquietud acerca del hecho de no contar con ciertas informaciones adicionales que creen pertinentes. Supongamos que en el problema de los empleos veraniegos, tales informaciones pueden consistir en hechos acerca de las neccsidades, flexibilidad o amistad de los posibles empleados, o de su deseo de trabajar. Pero, a pesar incluso de que no se disponga de esta información, tiende a resultar evidente que a la cliente del problema no le resultará de ninguna utilidad que los estudiantes decidan no responder a su petición de ayuda por la simple razón de 


\section{El problema de los empleos veraniegos}

El verano pasado, Maya inició un negocjo de concesión en el Parque de Recreo Wild Days. Sus vendedores ofrecen palomitas y bebidas por el parque, vendiendo en cualquier lugar donde puedan encontrar clientes. Maya necesita ayuda para decidir qué trabajadores va a volver a contratar el verano próximo.

El año pasado Maya contó con nueve vendedores. Este verano, solamente puede contar con seis -tres a tiempo completo y tres a mcdia jornada. Lo que pretende es volver a contratar a los vendedores que recauden más dinero, pero no sabe cómo compararles, puesto que trabajan un número diferente de horas. Además, los periodos en los que trabajan implican grandes diferencias. No en vano resuita más fácil vender en una noche del viernes con mucha gente que en una tarde lluviosa.

Maya repasó sus datos del año pasado. Para cada vendedor, calculó el número total de horas trabajadas y el dinero recolectado -cuando el negocio en el parque era alto (mucha gente), normal, y bajo (poca gente). (Véase la tabla.) Por favor, evalúe la efectividad en el negocio de cada vendedor del año pasado y decida qué tres vendedores đebería volver a contratar Maya a jornada completa y qué tres vendedores debería contratar a tiempo parcia!.

Escriba una carta a Maya con sus resultados. En dicha carta debe describir cómo ha evaluado a los vendedores. Incluya los detalles para que Maya pueda comprobar su trabajo y dé una explicación clara a fin de que pueda decidir si su método es bueno para que ella lo aplique.

Horas trabajadas el verano pasado

\begin{tabular}{|c|c|c|c|c|c|c|c|c|c|}
\hline & \multicolumn{3}{|c|}{ Junio } & \multicolumn{3}{|c|}{ Julio } & \multicolumn{3}{|c|}{ Agosto } \\
\hline & Lleno & Normal & Bajo & Lleno & Normal & Bajo & Lleno & Normal & Bajo \\
\hline MARIA & 12,5 & 15 & 9 & 10 & 14 & 17,5 & 12,5 & 33,5 & 3,5 \\
\hline KIM & 5,5 & 22 & 15.5 & 53,5 & 40 & 15.5 & 50 & 14 & 23,5 \\
\hline TERRY & 12 & 17 & 14,5 & 20 & 25 & 21,5 & 19,5 & 20,5 & 24,5 \\
\hline JOSE & 19,5 & 30,5 & 34 & 20 & 31 & 14 & 22 & 19,5 & 36 \\
\hline $\mathrm{CHAD}$ & 19,5 & .26 & 0 & 36 & 15,5 & 27 & 30 & 24 & 4,5 \\
\hline CHERI & 13 & 4,5 & 12 & 33,5 & 37.5 & 6,5 & 16 & 24 & 16,5 \\
\hline ROBIN & 26,5 & 43,5 & 27 & 67 & 26 & 3 & 41,5 & 58 & 5,5 \\
\hline TONY & 7,5 & 16 & 25 & -16. & 45,5 & 51 & 7.5 & 42 & 84 \\
\hline WILLY & 0 & 3 & 4,5 & 38 & 17,5 & 39 & 37 & 22 & 12 \\
\hline
\end{tabular}

Dinero recaudado el verano pasado (en dólares)

\begin{tabular}{|c|c|c|c|c|c|c|c|c|c|}
\hline & & & & & Julio & & & Agosto & \\
\hline & Lleno & Normal & Bajo & Lleno & Normat & Bajo & Llcno & Normal & Bajo \\
\hline MARIA & 690 & 780 & 452 & 699 & 758 & 835 & 788 & 1732 & 1462 \\
\hline KIM & 474 & 874 & 406 & 4612 & 2032 & 477 & 4500 & 834 & 712 \\
\hline TERRY & 1047 & 667 & 284 & 1389 & 804 & 450 & 1062 & 806 & 491 \\
\hline IOSE & 1263 & 1188 & 765 & I584 & 1668 & 449 & 1822 & 1276 & 1358 \\
\hline CHAD & 1264 & 1172 & 0 & 2477 & 681 & 548 & 1923 & 1130 & 89 \\
\hline CHERI & 1115 & 278 & 574 & 2972 & 2399 & 231 & 1322 & 1594 & 577 \\
\hline ROBIN & 2253 & 1702 & 610 & 4470 & 993 & 75 & 2754 & 2327 & 87 \\
\hline TONY & 550 & 903 & 928 & 1296 & 2360 & 2610 & 615 & 2184 & 2518 \\
\hline WILLY & 0 & 125 & 64 & 3073 & 767 & 768 & 3005 & 1253 & 253 \\
\hline
\end{tabular}


considerar que no disponen de cierta información pertinente.

Por consiguiente, inclusive en los primeros estadios de pensamiento acerca del problema, los estudiantes aceptan que, pura resolverlo, es necesario desarrollar una descripción simplificada (o modelo) que contemple las relaciones, pautas y tendencias significativas, y que simplifique la información, disponiéndola en una forma que resulte útil, y al mismo tiempo, evitando desviar la atención hatcia detalles superficiales de los datos o carentes de los mismos. Por lo tanto, estas finalidades tienden a aparecer reflejadas en las representaciones que formulan.

\section{Algunas caracteristicas de las interpretaciones inter-} medias de los estudiantes

Más adelante, los modos de pensamiento más elaborados tienden a ir más allá de la organización y proceso aislado de porciones de datos para centrarse en las relaciones, o en las pautas, o en las tendencias de los datos. Por ejemplo, en el problema de los empleos veraniegos, se tiende a dar mayor énfasis a las razones o a las tasas relacionadas con el tiempo y el dinero (p.e., dólares por hora); $y$, en general, se tiende a discernir entre los siguientes tipos de opciones. 1) Los estudiantes empiezan a calcular tasas dentro de cada categoría (lleno, normal, bajo; junio, julio, agosto). A continuación, puede que encuentren formas de acumulación de esas tasas. 2) Los estudiantes pueden empezar por calcular sumas o medias para cada categoría (lleno, normal, bajo; junio, julio, agosto). Después, quizá calcularán tasas basadas en estas acumulaciones. En cambio, al principio, los estudiantes podrían no haber conseguido distinguir entre a) calcular una media de varias tasas, y b) calcular una tasa individual basada en las medias de las recaudaciones y de los tiempos.

\section{Algunas características de las interpretaciones finales de los estudiantes}

Los resultados finales alcanzados por los estudiantes van, con frecuencia, más allá de las soluciones «estáticas" e incluyen declaraciones condicionales que contienen una diversidad de opciones o mecanismos que tienen en cuenta informaciones adicionales. Por ejemplo, en el problema de los empleos veraniegos, la solución dada puede permitir a los clientes asignar diferentes «pesos» que reflejen el criterio del cliente acerca de Ia relativa importancia de la información sobre los distintos meses o de los distintos periodos de trabajo. $O$ bien puede permitir al cliente ajustar pesos indicativos que se adaptan a sus propias preferencias, o utilizar procedimientos suplementarios, tales como entrevistas, para tener en cuenta informaciones adicionales, o considerar nuevas posibilidades de contratación que no habían sido consideradas cuándo se planteó el problema (tales como contratar a más o menos empleados a jornada completa o a tiempo parcial). Además, más que usar una sola regla que se aplica uniformemente a todos los posibles empleados, el procedimiento utilizado por los estudiantes puede contener una serie de procedimientos telescópi- cos. Pongamos por caso, para empezar, se puede utilizar un método con el fin de seleccionar empleados que estén en una categoría de cobligatorios»; y, a continuación, aplicar un procedimiento distinto para seleccionar empleados entre las restantes posibilidades. $O$, en vez de fiarse de las sumas o de las medias para simplificar la información, se pueden utilizar gráficos, como el que se presenta a continuacion, para destacar las tendencias.

En Ia búsqueda de soluciones de las actividades de obtención de estructuras, los procesos generales por los que pasan los estudiantes tienden a ser directos. Cuando se genera una descripción inicial, ésta puede comprender una combinación de términos orales, símbolos escrítos, dibujos o diagramas, o bien referencias a modelos concretos o a experiencias de la vida real. Pero, en todo caso, Ias representaciones tienden a organizar y a simplificar la situación que pueda observarse cierta información adicional, o que se pueda dirigir la atención hacia pautas o regularidades subyacentes que pueden, a su vez, forzar cambios conceptuales. Entonces, esta nueva información, con frecuencia, crea la necesidad de una descripción más refinada o más elaborada; y, esta nueva descripción, de nuevo, tiende a posibilitar una nueva ronda de información adicional que debe tomarse en consideración. Por consiguiente, los sistemas conceptuales internos y los sistemas de representación externos tienden ambos a ser inestables, a interactuar y a evolucionar continuamente; $y$, el ciclo general de desarrollo se repite hasta que la correspondencia entre el modelo y lo modelado aparece como suficientemente ajustada y suficientemente potente para producir los resultados deseados sin ulteriores adaptaciones.

Las observaciones anteriores conducen a un cierto número de corolarios acerca de las formas y funciones de las representaciones en el aprendizaje de las matemáticas y de la resolución de problemas. Por ejemplo, cuando una persona observa un sistema que otra persona ha construido, el sistema observado no es necesariamente idéntico al sistema construido. De hecho, incluso cuando Ios individuos observan sistemas que han construido ellos mismos, el sistema observado no es necesariamente idéntico al sistema construido. Hagamos una suposición, cuando un estudiante dibuja un diagrama de una situación compleja o cuando se describe dicha situación utilizando la lengua hablada o símbolos escritos, la persona que ha construido la descripción con frecuencia capta más información o diferente información que la que lee. Quizá, antes de haber generado la representación, la atención se había centrado en los detalles; pero, una vez que la representación está disponible, resultan aparentes, a menudo, nuevas pautas o regularidades. Las primeras conceptualizaciones de los estudiantes pueden no conseguir reconocer el clásico bosque tras los árboles, o viceversa; o, cuando centran su atención en un tipo de detalle, otros detalles pueden permanecer conceptualmente ignorados. Pero, de esta y de otras maneras, los significados, tanto de las estructuras como de Ios sistemas de representación, tienden a ser inestables; y esta falta de estabilidad, junto con las adaptaciones que se practican con el fin de incrementarla, son algunas de las fuerzas directrices más importantes que subyacen 
Tendencias de junio a julio y a agosto

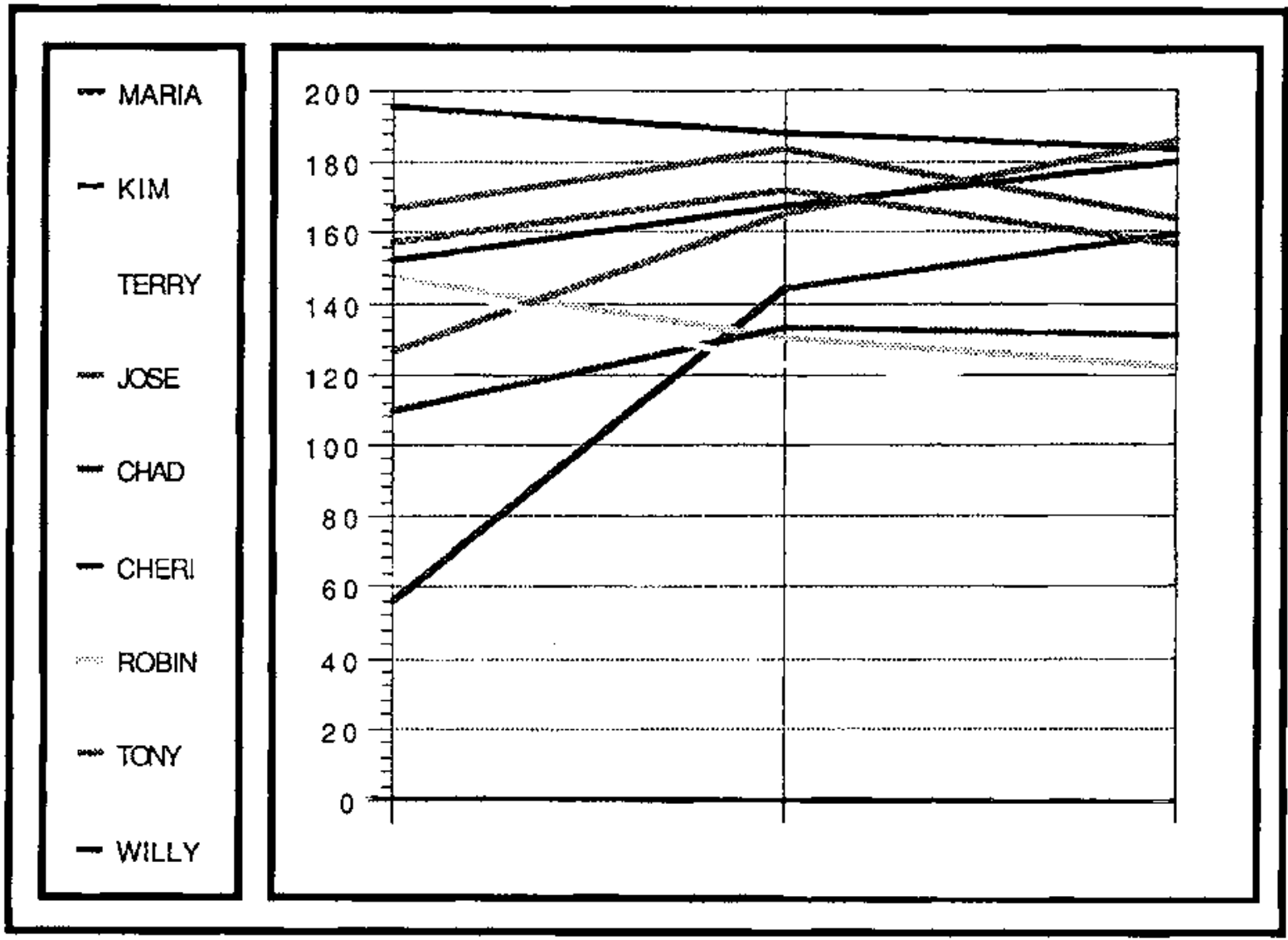

bajo el desarrollo de estructuras. Para los sistemas conceptuales, del mismo modo que para otros tipos de sistemas autoorganizados, «la supervivencia de lo estable" tiende a ser el análogo moderno más pertinente de la ley de Darwin sobre «la supervivencia del más dotadon.

\section{Las actividades de obtención de estructuras son sesiones de desarrollo conceptual local}

Cuando las actividades de obtención de estructuras implican sistemas conceptuales básicos (por ejemplo, fracciones, razones, tasas, proporciones u otros conceptos matemáticos elementales) que han sido estudiadas por psicólogos del desarrollo o por educadores matemáticos, el ciclo de modelización que siguen los estudiantes durante las sesiones de sesenta minutos de resolucion de problemas, a menudo, aparecen como ciclos locales o versiones localizadas de los estadios que los psicólogos del desarrollo han observado a lo largo de periodos de varios años. Al mismo tiempo, los procesos y mecanismos que contribuyen al desarrollo de estas estructuras tienden a ser los mismos que aquéllos que contribuyen al desarrollo conceptual a gran escala. En particular, el conflicto cognitivo, o la necesidad de desarrollar una mayor estabilidad conceptual, es un factor primario que crea la necesidad de una adaptación conceptual; y los sistemas de representación facilitan la progresiva diferenciación e integración de los sistemas conceptuales pertinentes.

En las actividades de obtención de estructuras en las que las soluciones normalmente implican una serie de ciclos interpretativos, dos de los más importantes fenómenos que requieren explicación son: a) “ ¿Cómo es que los estudiantes perciben la necesidad de un desarrollo más allá de sus conceptualizaciones iniciales sobre la situación problemática?»; $b$ ) « ¿Cómo es que son capaces de dirigir ese desarrollo hacia interpretaciones menos fútiles y deformadas?»

Para responder a la pregunta anterior, las teorías generadas por los psicólogos del desarrollo han demostrado resultar pertinentes, puesto que, cuando se interpretan las actividades de obtención de estructuras como actividades de desarrollo conceptual local (DCL): a) se pueden aplicar mecanismos que contribuyen al desarrollo conceptual general para explicar los procesos de resolución de problemas de los estudiantes (Lesh y Zawojewski, 1987); y b) se pueden aplicar mecanismos, importantes en las sesiones de desarrollo conceptual local, para 
Resolución de problemas aplicados $¥$ Actividades de obtención de estructuras

\section{La visión tradicional}

Se trata la resolución de problemas aplicados corno un caso especial de la resolución tradicional de problemas.

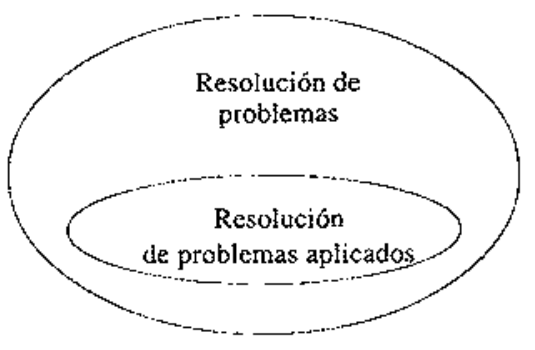

Si se considera la resolucion de problemas como ir de los datos a los objetivos, es lógico suponer lo siguiente:

* Los problemas aplicados (o actividades de análisis de estructuras) son casos especiales que implican datos "embatullados" y conocimientos del «mundo real», además del conocimiento necesario para situaciones más generales.

* I as herramientas heurísticas y otros mecanismos que se aplican a la resolución «general» de problemas debe particularizarse para resultar provechoso en situaciones de resolución de problemas aplicados.

Por consiguiente, sería de esperar que el aprendizaje de la resolución de problemas del «mundo real» comprendiera tres pasos:

* Primero, aprender tas ideas y destrezas básicas.

* A continuación, aprender algunos procesos y heurística de la resolución general de problemas (independientes del contenido).

* Por último (si el tiempo lo permite), aprender a utilizar las ideas, destrezas y herramientas heurísticas anteriores para situaciones en las que tamlisen șe requiere información adicional del «mundo real».

\section{La visión alternativa}

Se trata la resolución tradicional de problemas como un caso especial de actividades de obtención de estructuras.

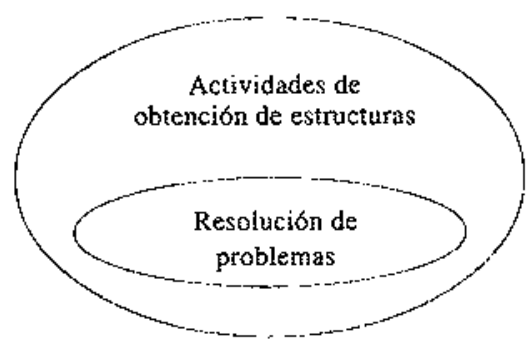

Si la característica esencial de la resolucion de problemas matemáticos comprende la interpretación de situaciones matemáticamente (modelizacion), los problemas no habituales comprenden más de un único ciclo de modelización y, si múltiples ciclos de modelización implican modos distintos de pensamiento acerca de los datos, objetivos o recorridos hacia la solución, es lógico suponer lo siguiente:

* La resolución tradicional de problemas es un caso especial en el que no se necesitan ciclos de modelización múltiples.

* Los procesos de solución implican mucho más que el procesado de la información mediante el uso de un único modelo invariante; también implican la transformación de modelos, ya que es el propio ciclo interpretativo el que se va modificando, ampliando, afinando.

* La construcción y afinamiento de modelos es lo mismo que di desamollo de estructuras; por tanto, las experiencias de resolución de problemas aplicados son importantes en el camino hacia el aprendizaje de las estructuras subyacentes. ayudar a explicar el desarrollo localizado de las capacidades generales de razonamiento de los estudiantes (Lesh y Kaput, 1988). Asimismo, es posible observar directamente estos mecanismos, puesto que, durante las actividades de obtención de estructuras, las pertinentes a menudo se modifican (se amplían, se afinan, se revisan), de forma significativa, en periodos de tiempo relativamente breves. Por consiguiente, conforme se amplían las estructuras pertinentes -se afinan, se establecen diferencias entre ellas, se integran o se adaptan de distintas muneras-, los investigadores pueden ir más allá de las descripciones de los sucesivos estadios de conocimiento para observar los mecanismos que promueven el desarrollo de un estadio a otro (Lesh, 1983). Adicionalmente, ya que es posible crear las condiciones que optimicen las posibilidades de que dicho desarrollo tenga lugar, sin dictar las direcciones que este desarrollo deba tomar, los investigadores en didáctica de las matemáticas han sido capaces de ir más allá de la investigación del típico desarrollo en entornos naturales para centrarse también en el desarrollo inducido, dentro de entornos meticulosamente controlados y matemáticamente enriquecidos (Lesh y Kelly, en prensa).

La aplicación de perspectivas desarrollistas a Ia resolución de problemas es un fenómeno relativamente nuevo en la investigación en didáctica de las matemáticas, en la que, tradicionalmente: a) Ia resolución de problemas se ha definido como ir de los datos a los objetivos cuando el recorrido no es inmediatamente obvio o estú bloquea$d o ;$ y b) la heurística se concibe como las respuestas a la pregunta: «¿Qué se puede hacer cuando se está bloqueado? Cuando se centra la atención en las actividades de obtención de estructuras, la esencia de la resolución de 
problemas implica encontrar formas de interpretar esas situaciones matemáticamente. Por consiguiente, en general, es más importante para los estudiantes encontrar formas de adaptar, modificar y afinar ideas que ya tienen, que intentar encontrar maneras de ser más eficiente cuando están bloqueados (por ejemplo, cuando no tienen ideas significativas o cuando los conceptos característicos no parecen pertinentes, tal como ocurre a menudo en acertijos y juegos). Por tanto, para desarrollar respuestas útiles a las actividades de obtención de estructuras, los tipos de herramientas heurísticas y las estrategias que resultan más útiles tienden a ser bastante distintas de aquéllos en los que se ha insistido en los problemas tradicionales, en los que las soluciones implican solamente un único ciclo interpretativo... y en los que pocas exigencias más se plantea a las capacidades de los estudiantes para introducir, modificar y adaptar las representaciones convenientes.

En las actividades de obtención de estructuras, y en otras situaciones de resolución de problemas que se centran en la generación de interpretaciones, el lenguaje, los símbolos, los gráficos y los esquemas organizativos que los estudiantes introducen tienden a ser descripciones parciales (simplificaciones) de sistemas externos. Sin embargo, puesto que estas descripciones se centran en relaciones, pautas y regularidades hipotéticas que se atribuyen a sistemas externos, más que como derivados de ellos, las representaciones son también externalizaciones de sistemas internos. Por consiguiente, las soluciones a tales problemas implican interacciones entre tres tipos de sistemas: a) sistemas conceptuales (internos) que residen en las mentes de los estudiantes; $b$ ) sistemas (externos) que se dan en la naturaleza o que tos construyen los humanos (pero que se construyeron como un fín en sí mismos, más que como representaciones para explicar otros sistemas); y c) modelos (externos) o sistemas de representación que funcionan como externalizaciones de sistemas conceptuales internos tanto como internalizaciones de sistemas externos.

\section{LOS HUMANOS CONTINUAMENTE PRO- YECTAMOS ESTRUCTURAS SOBRE LA REALIDAD}

AI hablar de sistemas que existen en el mundo real, algunos filósofos pueden pretender que las únicas pautas que perciben los estudiantes en estos sistemas son las que ellos mismos construyen (von Glasersfled, 1990); y hay una cierta verdad subyacente en este punto de vista. Pero, globalmente, resulta también simplista y desorientador. Por ejemplo:

* Una de las características más importantes de una era de la información es que las estructuras o sistemas conceptuales que hoy están en la mente humana pueden utilizarse para crear sistemas que funcionan como objetos concretos en el mundo del mañana. Y sistemas que se crean como un fin en sí mismos hoy pueden utilizarse de forma simbólica para entender otros sistemas del mañana. Por ejemplo, cuando un director de una empresa

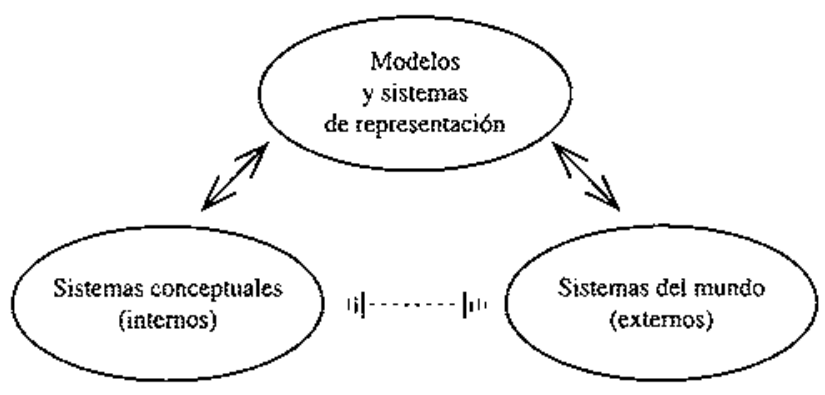

utiliza una calculadora gráfica (o una hoja de cálculo con gráficos) para hacer previsiones acerca de la maximización de las tendencias coste-beneficio, estas herramientas le permiten crear sistemas empresariales de un tipo completamente nuevo que no existían antes de que esas herramientas estuvieran disponibles y, asimismo, amplían las capacidades conceptuales y procedimentales del director a la hora de tratar estos sistemas externos recién introducidos. Como consecuencia, entre los «objetos» matemáticos que influyen sobre nuestras vidas cotidianas, muchos de los más importantes son sistemas -sistemas complejos, dinámicos e interactivos-que pueden variar en cuanto a su alcance, desde sistemas económicos y de comunicaciones a gran escala hasta sistemas a pequeña escaia de actividades cotidianas de planificación del tiempo, de organización o de contabilidad.

* Otra importante característica de una sociedad tecnificada es que los humanos sabemos cómo crear sistemas que nosotros mismos no sabemos cómo describir, explicar, predecir o controlar. Ésta es la razón por la que algunos de los más importantes objetivos de la enseñanza de las matemáticas consisten en ayudar a los estudiantes a desarrollar estructuras potentes o «tecnologías conceptuales» para entender esos sistemas. A veces, estas tecnologías conceptuales solamente implican soportes de representación primitivos; pero, en otros casos, implican hojas de cálculo, gráficos, curvas, programas u otros sistemas de representación que residen en calculadoras, ordenadores u otros mecanismos técnicos; o bien, implican «herramientas inteligentes» basadas en las coordenadas cartesianas, matrices, espacios vectoriales, diagramas complejos u otros sistemas simbólicos que exigen el uso de la lengua hablada, de símbolos escritos o de otros medios de representación.

En cualquiera de los casos anteriores, los humanos proyectamos nuestras estructuras continuamente sobre la realidad, en forma de artefactos que no se utilizan con fines de representación, y también en forma de sistemas de representación y otras tecnologías conceptuales.

\section{LAS REPRESENTACIONES PUEDEN CONSIDERARSE COMO TECNOLOGIAS CONCEPTUALES O «HERRAMIENTAS IN- TELIGENTES»}

Considerar los sistemas de representación como therramientas inteligentes» tiene una serie de implicaciones 
beneficiosas: a) Aunque hay una razón por la cual resulta natural hablar de estructuras matemáticas como si fueran puros sistemas desligados de cualquier herramienta, representación o artefacto externo, en la práctica, a parte de situaciones triviales, estos sistemas conceptuales rara vez funcionan sin el soporte de potentes herramientas en las que están (parcialmente) contenidos. b) Cuando las herramientas inteligentes comprenden ordenadores, caIculadoras u otros amplificadores conceptuales, es evidente que los sistemas conceptuales de sus usuarios no residen únicamente dentro de las fronteras de sus mentes. Su inteligencia, sus capacidades de conceptualización y de proceso se distribuyen a lo largo de una diversidad de niveles y tipos de herramientas y representaciones. c) Algunas herramientas son claramente más potentes que otras y algunas de ellas tienden a resultar las más útiles para según qué propósitos. Por ejemplo, hace muchos años, Bruner (1973) destacó el importante papel que desempeñan los sistemas de representación como medio de ayuda al hombre para ir más allá de la información que se le proporciona en situaciones concretas de aprendizaje o de resolucion de problemas. Sin embargo, si no se pide nunca a los estudiantes que desarrollen herramientas «re-utilizables» o compartibles o que puedan ser modificadas fácilmente para ser aplicadas a otras situaciones, no deberíamos sorprendernos de que a sus estructuras les falte generalidad, portabilidad y modificabilidad. d) Construir una herramienta general no garantiza que su constructor sea capaz de generalizar.

Por otro lado, es bien sabido que, cuando una persona sostiene un martillo en la mano, todo parece tender a tener el aspecto de un clavo. Por tanto, respecto a las herramientas, el problema clave del aprendizaje no es necesariamente la capacitación para generalizar. A menudo, el problema clave es que los estudiantes sean más capaces de discernir e identificar las situaciones en las cuales una herramienta resulta aplicable.

\section{REFERENCIAS BIBLIOGRÁFICAS}

BRUNER, J. (1973). Beyond the information given. Nueva York: Noiton \& Co.

DISESSA, A.A., HAMMER, F., SHERIN, B. y KOLPAKOWSKI, T. (1991). Inventing graphing: Meta-representational expertise in children. Journal of Mathematical Behaviour, 10(2), pp. $117-160$.
Cuando se formulan los problemas de tal manera que los estudiantes necesitan construir descripciones, explicaciones y justificaciones a propósito, tienden a introducir en sus soluciones y procesos hacia dichas soluciones una gama mucho más amplia de representaciones. Adicionalmente, cuando queda clara la finalidad de una descripción, los estudiantes se sienten inclinados a abordar los temas de representación relacionados con los niveles adecuados de precisión, exactitud, puntualidad, etc. Por consiguiente, cuando se facilita una descripción inicial, casi siempre es necesario ampliarla, afinarla o revisarla con el fin de hacerla suficientemente aplicable. Por tanto, las soluciones implican una serie de ciclos de afinamiento de la representación.

* Cada vez hay más «herramientas inteligentes» disponibles (tales como calculadoras, hojas de cálculo o programas de gráficos). Por esa razón: a) la interpretación del problema resulta, a menudo, problemática debido a que es necesario convertir la situación a una forma que facilite el uso de una de esas herramientas; y $b$ ) Ja interpretación de la respuesta resulta problemática debido a que los resultados que genera la herramienta pueden no estar en una forma inmediatamente utilizable. Así, puede ser necesario convertir los decimales en fracciones, o la información gráfica en información numérica.

* Las capacidades matemáticas en las que se hace hincapié, a menudo, insisten en la comunicación, Ia planificación, el seguimiento y otros tipos de pensamiento de alto rango que reclaman capacidades de representación ( $\mathrm{Di}$ Sessa, 1996); es decir, que los estudiantes tienen que ir más allá de pensar con una representación matemática dada para pensar además acerca de la potencia o debilidad relativa de las representaciones alternativas.

\section{NOTA}

Este artículo ha sido traducido del inglés por J. Tortella.
KATIMS, N. y LESH, R. (1994). Packets: A Guidebook for inservice Mathematics Teacher Development. D.C. Heath, Lexington, MA.

KELLY, E. y LESH, R. (eds.) (en prensa). Innovative Research Design in Mathematics \& Science Education. (Manuscrito disponible por parte de los eds. Editorial todavía por determinar). 
LESH, R. y KAPUT, J. (1988). Interpreting modeling as local conceptual development, en DeLange, J. y Doorman, $M$. (eds.). Senior Secondary Mathematics Education. Utrecht, Holanda: OW\&OC.

LESH, R. y KOLPAKOWSKI, T. (1987). Problem solving, en Post, T. (ed.). Teaching Mathematics in Grades $K-8$ : Research-based Methods. Boston: Allyn \& Bacon.

LESH, R., AMIT, M., y SCHORR, R. (en prensa). Six Principles For Writing Effective Model-Eliciting Activities, en Gal, I. y Garfield, J. Research on Statistical Literacy. Philadelphia: Franklin Institute.

LESH, R., HOOVER, M. y KELLY, A. (1993). Equity, Technology and Teacher Development, en Wirszup, I. y Streit, R. (eds.). Developments on School Mathematics Education Around the World, Vol. 3. National Council of Teachers of Mathematics, Reston, VA.

SCHANK, R.C. (1990).Tell Me A Story: A New Look At Real and Artificial memory. Nueva York: Charles Scribner's Sons, MacMillan.

VON GLASERSFELD, E. (1990). An Exposition of Constructivism: Why Some Like It Radical, en Davis, R.B., Maher, C.A. y Noddings, N. (eds.). Constructivist Views on the Teaching and Leaming of Mathematics, National Council of Teachers of Mathematics, Reston, VA., pp. $107-122$. 
, 